IVth Conf. on the Intersections Between Particle and Nuclear Physics,

Conf $9105106-1$ Tucson, Arizona, 5/24-29/91.

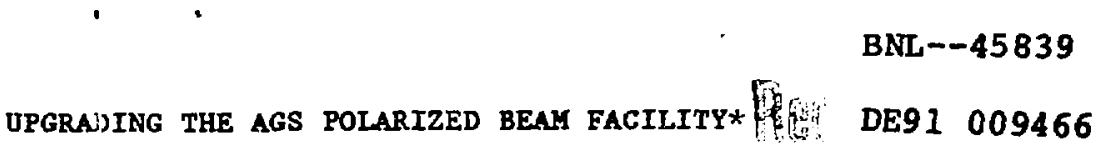

L.G. Ratner

AGS Department, Brookhaven National Laboratory, Upton, New York 11973

\title{
ABSTRACT
}

Although present techniques for crossing depolarizing resonances in circular accelerators work, they are very time-consuming to implement and were only able to provide about a $40 \%$ polarized beam at $22 \mathrm{GeV}$ in the Alternating Gradient Synchrotron (AGS). We propose to install a partial "Siberian Snake" solenoid in the AGS to eliminate the need to correct imperfection resonances and to make other modifications in our intrinsic resonance correctors. This will allow us to reach an energy of $25 \mathrm{GeV}$ with $70 \%$ polarization and will enable the AGS to be an efficient injector of polarized protons into the Relativistic Heavy Ion Collider (RHIC), as well as being able to carry on a fixed-target program with minimum set-up time.

\section{SIBERIAN SNAKES}

Recent tests ${ }^{1}$ at the Indiana University Cyclotron Facility (IUCF) have given experimental proof that Siberian Snakes (invented by Ya.S. Derbenev and A.M. Kondratenko) ${ }^{2}$ can indeed overcome the effects of depolarizing resonances in circular accelerators. The basic idea of these devices is to rotate the spin of a circulating proton as it traverses the snake by $180^{\circ}$ around a horizontal axis. Thus, any effects of depolarizing resonances will be cancelled on successive turns through the snake. Snakes made up of a series of dipole magnets that deflect the beam horizontally and vertically in alternate magnets work well at high energies $(\geq 20 \mathrm{GeV})$, but lead to too large an orbit deflection and too long a magnet length at low energies. Therefore, a solenoid spin rotator which does not distort orbits and is a reasonable length at low energies was used in the IUCF. As shown in Figures 1,2 and 3 , this snake was able to preserve polarization in the presence of imperfection, intrinsic, and synchrotron resonances. The most important point though for low energy accelerators, where there is very little available straight section length, is shown in Figure 1, where the snake excitation can be reduced from $100 \%$ to a few percent and still prevent depolarization from imperfection resonances. These resonances, due to vertical closed orbit distortions, occur about every $1 / 2 \mathrm{GeV}$ and change with time. They are sensitive to a few mils change in vertical position of the accelerator and thus may even change over a running period. The elimination of their effect charges a 2-3 week tune-up time to a 2-3 day effort.

*Work performed under the auspices of the U.S. Department of Energy.

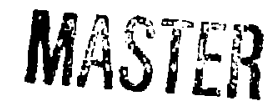




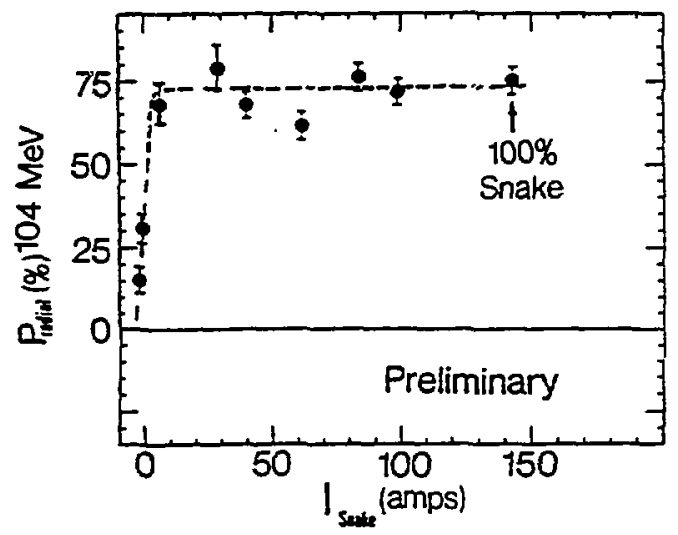

Fig. 1. A partial Snake overcoming the $G_{\gamma}-2$ imperfection depolarizing resonance.

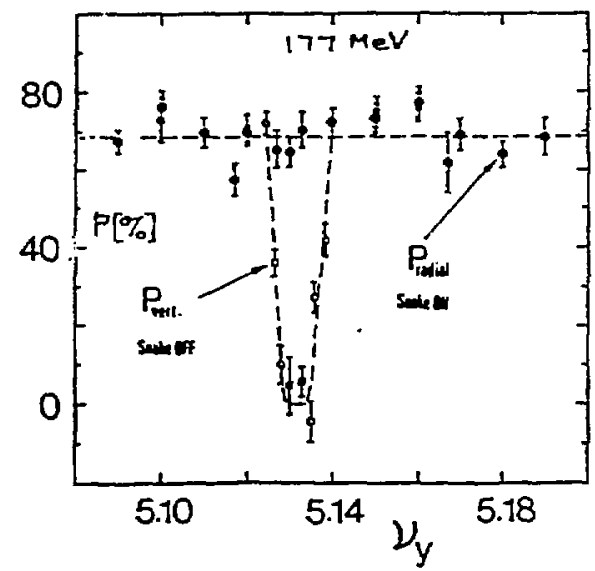

Fig. 2. The Siberian Snake overcoming the $\mathrm{G} \gamma$ $-3+\nu_{y}$ intrinsic depolarizing resonance.

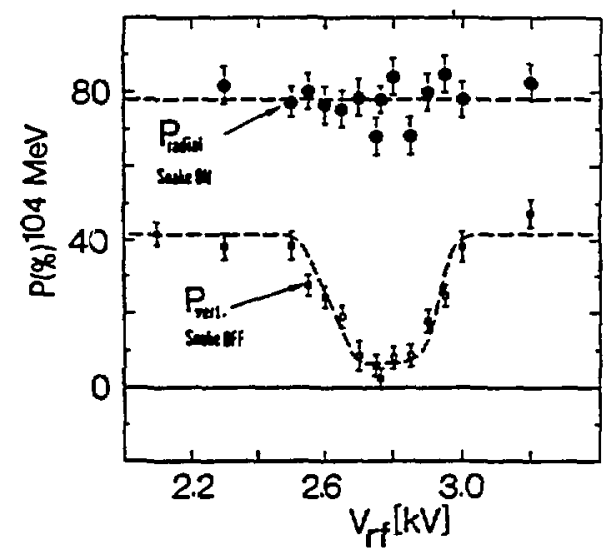

Fig. 3. The Snake overcoming a synchrotron depolarizing resonance.

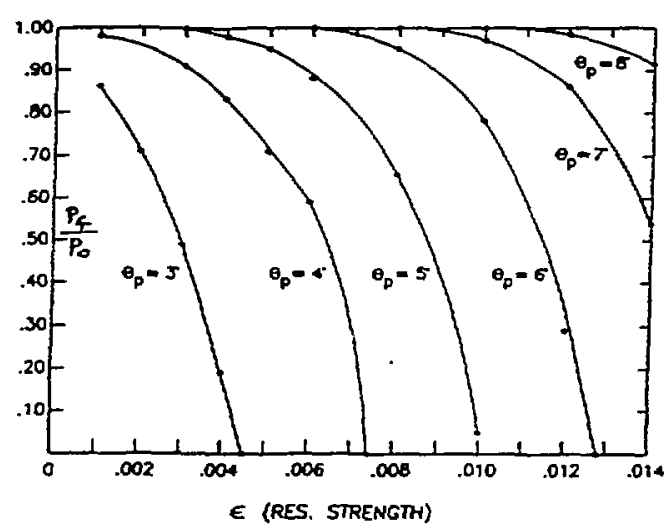

Fig. 4. Resonance strengths vs. polarization survival $\left(\mathrm{P}^{\mathrm{f}} / \mathrm{P}^{\mathrm{O}}\right)$ from computer simulations for several different strength snakes. 
The longest straight section in the AGS is 10 feet long. We, therefore, considered the maximum size snake that we could fit into the machine. Mecharical considerations of ends, flanges, etc., led us to decide that a $90^{\prime \prime}$ effective length would be maximum. Figure 4 shows a graph of resonance strength versus polarization survival for a snake of various precession angles $(\theta \mathrm{p}) . \theta \mathrm{p}=180^{\circ}$ is a $100 \%$ snake. This graph was generated by computer simulations for the AGS. Our next step was to determine the strength of the AGS resonances from our actual runs with polarized beam and see what strength snake would be needed. This is summarized in Table $I$. Choosing $a^{\circ}$ (5x) snake gives $100 \mathrm{z}$ polarization survival at $24.8 \mathrm{GeV} / \mathrm{c}$ and requires an $\int \mathrm{Bdl}-4.66 \mathrm{~T}-\mathrm{M}$. In Figure 6 , we show a conceptual coil design using $12.6 \mathrm{kA}$ at about $200 \mathrm{~V}$ for a ramped $6 \mathrm{n}$ i.d. room-temperature solenoid which fulfills the field requirements. Despite the large current, the ramped mode gives an RMS power of only $92 \mathrm{~kW}$ which can be easily dissipated with water-cooled coils.

Table I. $P_{f} / P_{o}$ for AGS Resonances at Several Snake Precession Angles

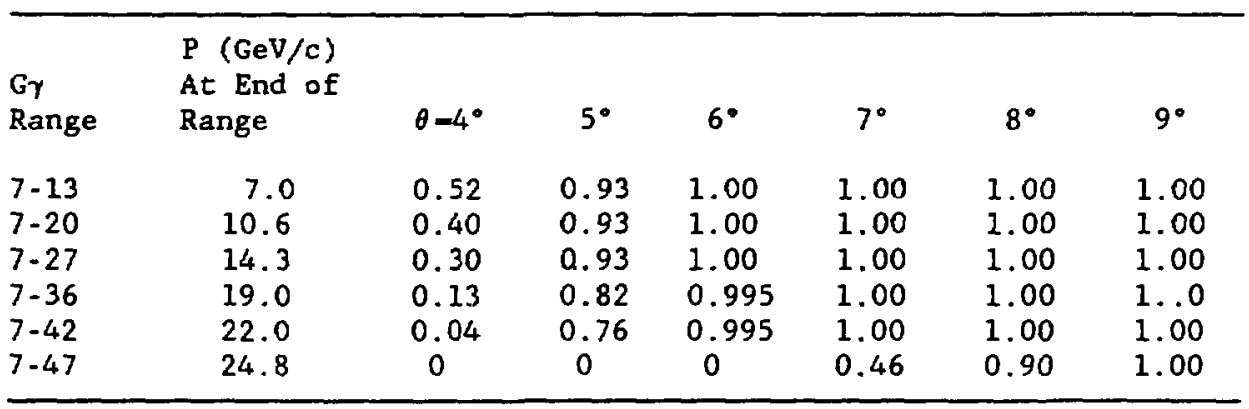

COIL CROSS-SECTION

4 LAYERS, SHOWING ONE OF 76 TURNS NOT TO SCNE

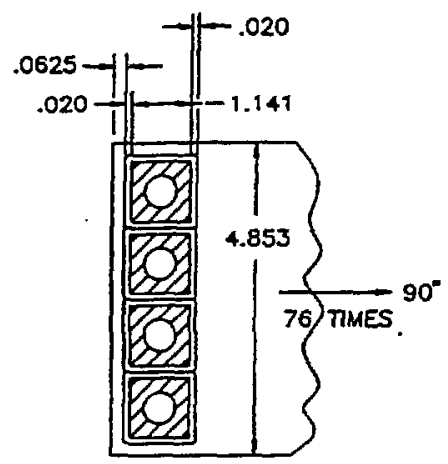

SOLENOID END VEW

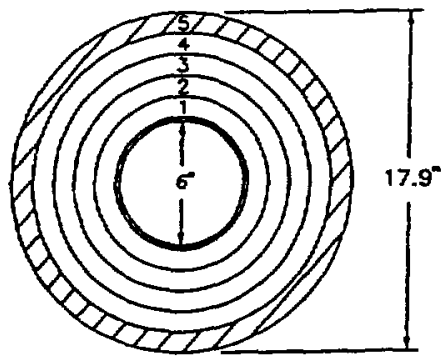

11. 2. 13. 14 LAYERS OF COPPER I5 1" Fe RETURN PATH $B_{\mathrm{Fe}} \sim 11 \mathrm{Kg}$

Fig. 5. Schematic of coil and end view--not to scale. 


\section{OTHER CORRECTORS}

The original AGS fast tune shift quadrupoles were configured to reach $22 \mathrm{GeV} / \mathrm{c}$. To go to $24.8 \mathrm{GeV} / \mathrm{c}$ (needed for RHIC), the power supplies for these 10 quadrupoles must be rearranged so that an additional high current supply is made available for the $G \gamma=36+\nu_{y}$ intrinsic resonance crossing. In addition, it is necessary in the AGS to also have a slow tune shift of the order of $\Delta \nu_{y}= \pm 0.15$ in order to move the machine tune away from the direction of the fast tune jump. Thus, a $\Delta v=+0.3$ from the fast quads can start at $\nu_{y}$ 0.15 and go to $\nu_{y}+0.15$ and we do not exceed a machine tune limit of $\Delta \nu_{y}= \pm 0.2$, but we still move the tune rapidly by 0.3 . To perform this function, there are 12 vertical tune quads, one in each superperiod, which will have to be energized to give optimal corrections for the intrinsic resonances.

\section{RHIC INJECTOR}

The above program should provide a polarized beam from the AGS which can be transported to RHIC without loss of polarization and with an intensity which gives the same luminosity as the unpolarized beam. A $25 \mathrm{GeV}$ polarimeter will be needed in the transfer line and probably a Coulomb interference polarimeter in RHIC to measure the high energy polarization. A preliminary study of RHIC requirements ${ }^{3}$ indicates that to polarize RHIC will require about $\$ 5 \mathrm{M}$, but there are no apparent technical probleas.

\section{REFERENCES}

1. A.D. Krisch, et al., Phys. Rev Lett. 63,1137 (1989). J.E. Goodwin, et al., Phys. Rev, Lett. 64, 2779 (1990).

2. Ya.S. Derbenev and A.M. Kondratenko, Sov. Phys. JETP 35,230 (1972).

3. L.G. Ratner, private communication. 